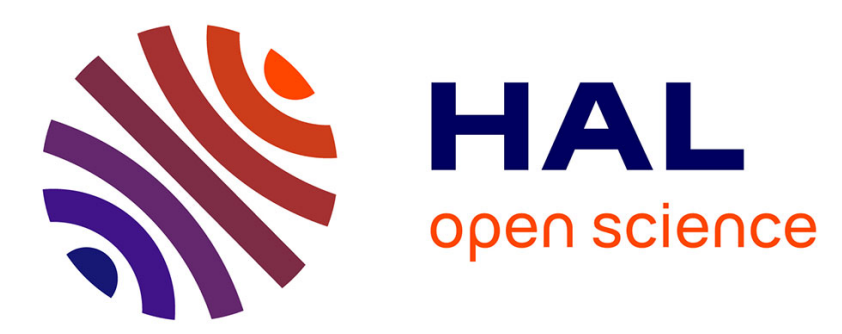

\title{
Airfoil Tip Leakage Aeroacoustics Predictions using a Lattice Boltzmann Based Method
}

Adrian Mann, Min-Suk Kim, Jingshu Wu, Franck Pérot, Julien Grilliat, Marc C. Jacob, Miles Colman

\section{- To cite this version:}

Adrian Mann, Min-Suk Kim, Jingshu Wu, Franck Pérot, Julien Grilliat, et al.. Airfoil Tip Leakage Aeroacoustics Predictions using a Lattice Boltzmann Based Method. 22nd AIAA/CEAS Aeroacoustics Conference, May 2016, Lyon, France. pp.1-17, 10.2514/6.2016-2825 . hal-02177161

\section{HAL Id: hal-02177161 https://hal.science/hal-02177161}

Submitted on 8 Jul 2019

HAL is a multi-disciplinary open access archive for the deposit and dissemination of scientific research documents, whether they are published or not. The documents may come from teaching and research institutions in France or abroad, or from public or private research centers.
L'archive ouverte pluridisciplinaire HAL, est destinée au dépôt et à la diffusion de documents scientifiques de niveau recherche, publiés ou non, émanant des établissements d'enseignement et de recherche français ou étrangers, des laboratoires publics ou privés. 


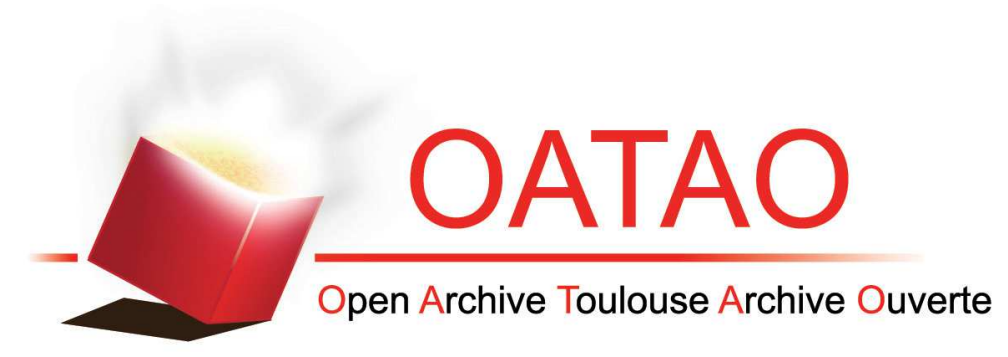

\section{Open Archive Toulouse Archive Ouverte (OATAO)}

OATAO is an open access repository that collects the work of some Toulouse researchers and makes it freely available over the web where possible.

This is an author's version published in: https://oatao.univ-toulouse.fr/24061

Official URL : https://doi.org/10.2514/6.2016-2825

\section{To cite this version :}

Mann, Adrian and Kim, Min-Suk and Wu, Jingshu and Pérot, Franck and Grilliat, Julien and Jacob, Marc C. and Colman, Miles Airfoil Tip Leakage Aeroacoustics Predictions using a Lattice Boltzmann Based Method. (2016) In: 22nd AIAA/CEAS Aeroacoustics Conference, 30 May 2016 - 1 June 2016 (Lyon, France).

Any correspondence concerning this service should be sent to the repository administrator: tech-oatao@listes-diff.inp-toulouse.fr 


\title{
Airfoil Tip Leakage Aeroacoustics Predictions using a Lattice Boltzmann Based Method
}

\author{
Adrien Mann ${ }^{1}$, Min-Suk Kim², Jingshu $\mathrm{Wu}^{3}$, and Franck Pérot ${ }^{4}$ \\ Exa Corporation \\ 150 North Hill Drive, Brisbane, CA 94005, USA \\ Julien Grilliat ${ }^{5}$ \\ ebm-papst St. Georgen GmbH \& Co. KG \\ Hermann-Papst-Straße 1, 78112 St. Georgen, Germany \\ Marc C. Jacob 6 \\ Laboratoire de Mécanique des Fluides et d'Acoustique UMR CNRS 5509, \\ Ecole Centrale de Lyon/ Université de Lyon, Ecully, France \\ also at ISAE-SupAéro, Toulouse, France \\ Miles Colman ${ }^{7}$ \\ Kitty Hawk Corp. \\ 2639 Terminal Blvd., Mountain View, CA 94043, USA
}

\begin{abstract}
The noise produced by rotating systems such as fans and turbo machines is of growing importance in the academic and engineering communities. The prediction and understanding of the physical mechanisms associated with noise generation are required in order to develop innovative solutions able to efficiently reduce radiated acoustics levels. The flow-induced noise generation mechanisms related to rotating devices are various and complex, and one of them is related to the blade tip flow. The tip flow noise, or tip leakage noise, is particularly important for free-tip configurations, for which the tip flow induced by the pressure gradient between the suction and pressure sides can be particularly intense. The experimental investigation of this mechanism is practically challenging. Consequently, a simplified non-rotating representative configuration has been proposed, and has been previously investigated experimentally. In this paper, transient, compressible, and time-explicit Computational Fluid Dynamics/Computational Aero-Acoustics (CFD/CAA) simulations of an airfoil tip leakage flow for this simplified geometry are performed using a Lattice Boltzmann Method (LBM) based approach. The studied configuration is a NACA 5510 airfoil profile at high Reynolds number flow conditions, for which a variable size gap is introduced between the airfoil and one of the end plates, modeling the tip gap encountered in free-tip fans. First, the simulation results are compared with experimental results to validate the numerical approach. Further investigation of the numerical results underlines the connection between the tip vortex structures and noise radiation, including a parametric study on the Angle of Attack (AoA) and the tip gap width.
\end{abstract}

\footnotetext{
${ }^{1}$ Principal Application Engineer, Aeroacoustics Applications, amann@exa.com

${ }^{2}$ Principal Application Engineer, Aeroacoustics Applications, $\underline{\text { mskim@exa.com }}$

${ }^{3}$ Principal Application Engineer, HVNA Group, jingshu@exa.com

${ }^{4}$ Senior Director, Aeroacoustics Applications, perot@exa.com

${ }^{5}$ Head of Aerodynamics, Acoustics and Vibrations Labs, julien.grilliat@ de.ebmpapst.com

6 Professor, Laboratoire de Mécanique des Fluides et d'Acoustique, Université de Lyon, Département d'Aérodynamique, Energétique et Propulsion (ISAE), AIAA Member, marc.jacob@ec-lyon.fr

${ }^{7}$ Mechanical Engineer, mcolman@gmail.com
} 


\section{Introduction}

$\mathrm{T}$ he noise generated by fans and turbo machinery has long been of interest to the community. Rising consumer expectations and recent regulations have made fan noise analysis and mitigation a more important part of fan integration for vehicles, aircrafts, HVAC systems and heavy industry equipment. Fan noise can be measured in experimental tests, but experiments require physical prototypes and have turn-around times which do not satisfy production constraints. In addition, the scale of some of the flow structures responsible for noise generation requires advanced experimental equipment to detect them, which are costly and often difficult to set up properly. Validated simulation capabilities for fan noise, able to simultaneously evaluate both airflow and acoustic performance, represent an attractive alternative enabling rapid evaluation, design optimization work and insight into and visualization of the sources of noise.

To isolate the main source of unsteady flow structures and noise in fan blades, a simpler experimental case that has been well-studied and possesses qualitatively similar dimensions to a fan blade is examined. The simulation results reported in the present work are based on the experimental study of an airfoil with tip gap flow in a quasi-2D jet with a free stream velocity $U_{0}^{1-4}$. At the exit of the wind tunnel, the airfoil is mounted between two end plates. A variable gap between the tunnel wall and the airfoil is introduced. The highly loaded airfoil with tip gap generates a tip vortex via the same mechanism as a rotating fan blade operating near a stationary wall, although no moving parts are involved in this particular setup.

In this study, the Computational Fluid Dynamics/Computational AeroAcoustics (CFD/CAA) solver PowerFLOW based on the Lattice-Boltzmann Method is used to predict the transient flow field and corresponding flow-induced noise radiation ${ }^{5-12}$. The solver has been successfully applied in numerous industrial analyses, academic validations and investigations such as wind-noise, HVAC, fans, blower and wind-turbine problems ${ }^{13-24}$. Wall Pressure Fluctuations (WPF) near the tip gap are the focus of this paper, as the noise-generating mechanisms of interest for fans are expected to correlate to the measurement locations on the blade.

The paper is organized in the following sections. The experimental and numerical setups are presented in section II, including a wind-tunnel flow quality study. Section III presents a study of the accuracy of the numerical method via the correlation of the simulated mean/transient flow field results with the tests ${ }^{1-4}$, as well as an in-depth analysis of the flow topology and its correlation to noise generation. In order to gain deeper insights in the noise-generating mechanisms occurring in the vicinity of the blade tip, two parametric studies, based on the Angle of Attack (AoA) and the tip gap width, are provided with the corresponding analysis in the final section IV.

\section{Experimental and numerical setups}

\section{II.A. Experimental setup}

The tested geometry is shown in Figure 1 and corresponds to a NACA 5510 airfoil (10\% thickness, 5\% camber) with a $200 \mathrm{~mm}$ chord and a $200 \mathrm{~mm}$ span. The angle of attack of the airfoil can be varied from $0^{\circ}$ to $20^{\circ}$ by rotating the wooden plates, and the tip gap width $\Delta z$ between the wall and the airfoil can be adjusted from $\Delta z=0 \mathrm{~mm}$ to $\Delta z=200$ $\mathrm{mm}$, corresponding to the distance between the end plates.

The baseline configuration has the following properties: $A o A=15^{\circ}, \Delta z=10 \mathrm{~mm}, U_{0}=70 \mathrm{~m} / \mathrm{s}$. The corresponding Reynolds number based on the chord is $\operatorname{Re}_{\mathrm{c}}=9.6 \cdot 10^{5}$. This set of parameters has been chosen because such a setup best represents a typical configuration for axial cooling fans and rotating machinery.

The available measurements in Grilliat ${ }^{1}$ are:

- Hot Wire Anemometry (HWA): turbulent boundary layer and velocity profiles at various locations

- Time-average pressure coefficient $C_{P}$ at the surface of the airfoil and endplates

- Power Spectrum Density (PSD) of Wall Pressure Fluctuations (WPF) on the airfoil and end plates

- Laser Particle Image Velocimetry (PIV) measurements in various planes around the airfoil parallel to the end plates 

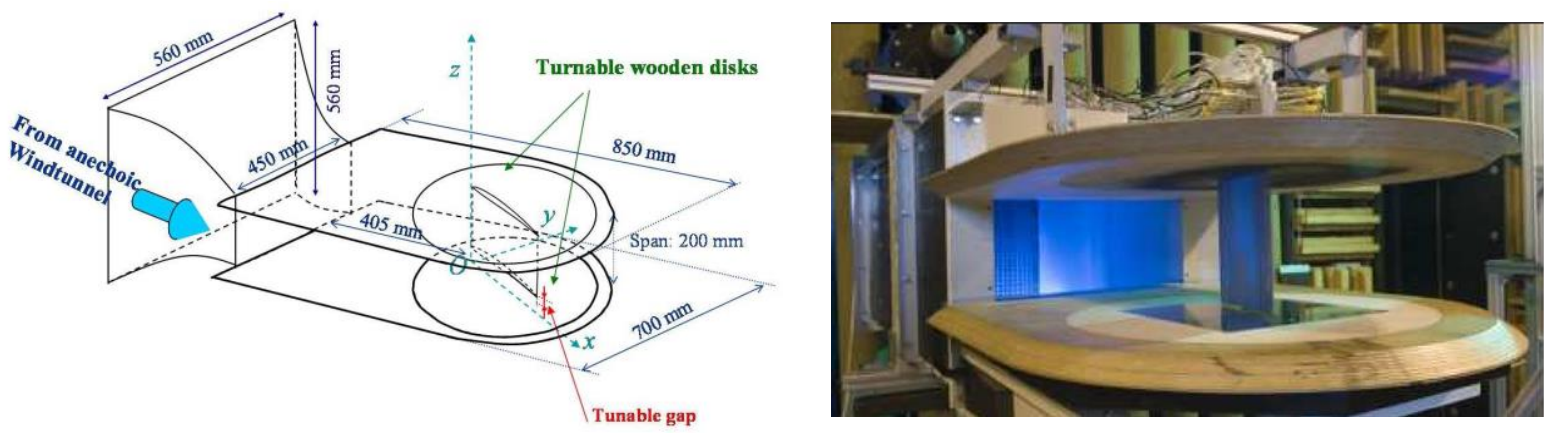

Figure 1. Experimental setup and geometry ${ }^{2}$.

The pressure measurement locations on the airfoil are shown in Figure 2. Pressure taps from \#19 to \#46 are located on the airfoil and taps A and B are located on the side of the airfoil, facing the tip gap. Taps \#49 to \#53 are placed on the lower plate and face the gap. The pressure fluctuations are measured using remote microphones and the obtained PSD are considered by the authors to be accurate up to $6-8 \mathrm{kHz}^{1}$. Consequently, comparison to experiment will be presented between $100 \mathrm{~Hz}$ and $5 \mathrm{kHz}$. Taps \#1-13, not shown in Figure 2, are located on the airfoil centerline and provide mid-span data. Taps \#1-13,\#18-21, \#22,\#29 and all taps located $1.5 \mathrm{~mm}$ from the tip gap are also instrumented to measure the mean pressure and used to characterize the pressure coefficient along the chord.

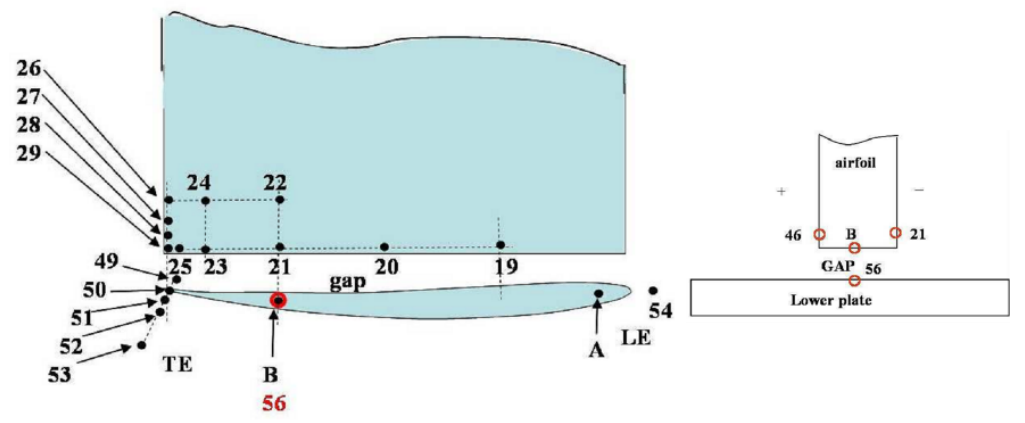

Figure 2. Pressure measurement locations ${ }^{2}$.

The boundary layer thickness $\delta$ of the incoming flow on the baseline configuration is characterized with HWA half a chord upstream of the airfoil. The reported thickness is $\delta=18 \mathrm{~mm}^{1}$.

\section{II.B. Simulation method}

PowerFLOW 5.3 based on the Lattice Boltzmann Method (LBM) is used to calculate unsteady flow physics and the corresponding flow-induced noise generation and radiation. Lattice-based methods are by nature explicit, transient and compressible, and are an alternative to traditional CFD methods based on the discretization of the Navier-Stokes equations and derived variations $5,6,12,27,30,33$. The basic idea of LBM is to track the advection and collisions of fluid particles. Since the average number of particles in a representative volume of fluid far exceeds the computing power required to track them individually, the particles are grouped into an integer number of discrete directions with index $i$. The computation follows the particle distribution function $f_{i}$ which represents the number of particles per unit of volume, also called voxel, at a specific time and location moving with velocity $c_{i}$. As in statistical physics, the flow variables such as density and velocity are determined by taking the appropriate moments, i.e. summations over the set of discrete directions, of the particle distribution function ${ }^{8-10,25,26,28,29,31,32}$. LBM is used and validated across many aeroacoustics applications such as automotive wind noise ${ }^{13}$, acoustics propagation ${ }^{14}$, airframe noise ${ }^{15}$, sunroof buffeting ${ }^{16}$, exhaust systems ${ }^{17}$, fan noise ${ }^{18-21}$ and HVAC systems noise ${ }^{22-24}$. Further details on LBM can be found in the cited references.

The geometry used in the simulation is shown below in Figure 3, corresponding to the complete real 3D geometry presented in the previous section. The main differences with the experimental setup are corresponding to the inlet portion of the simulated geometry for which the full experimental geometry was not available, and the bevel angle on the endplate edges, whose exact value is unknown. Also, the jet catcher of the experiment is not present in the 
simulation. In order to match the measured boundary layer thickness $\delta$, a frictionless wall condition is used over a $d=255 \mathrm{~mm}$ distance from the inlet of the simulation domain. Further downstream, a smooth wall boundary condition is used, allowing the development of a turbulent boundary layer inside the rectangular tunnel section. The mean flow velocity $U_{0}$ is imposed at the inlet of the domain, which is represented with a red area in Figure 3 . The airfoil and the plates are inside a large volume of fluid with multiple sponge zones to create an anechoic chamber. For the baseline case, the finest voxel length of $0.25 \mathrm{~mm}$ gives 40 voxels across the tip gap. A total of 10 probes located on an arc 1.7 $\mathrm{m}$ away from the airfoil centerline, as presented in Figure 4, are used to directly measure the far-field noise generated by the system.
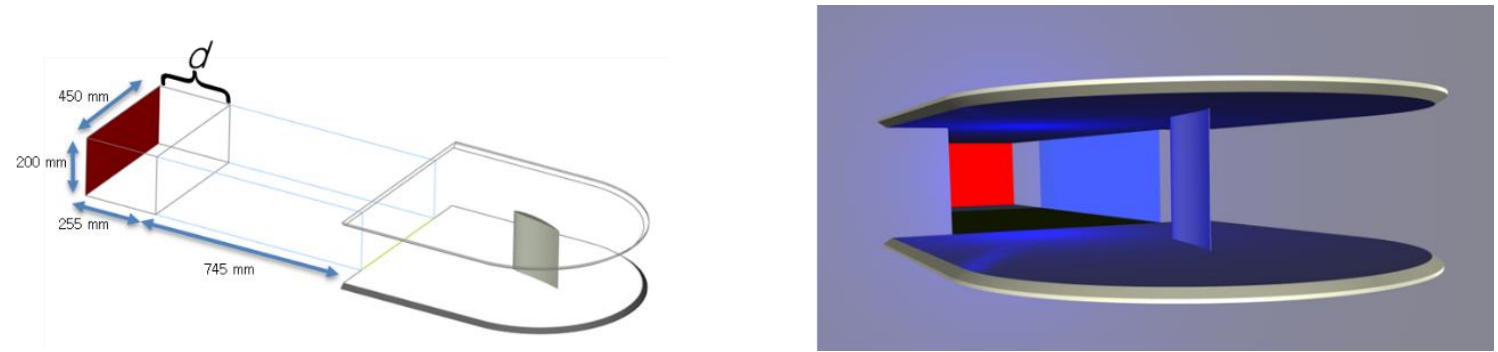

Figure 3. Simulation domain and boundary conditions.

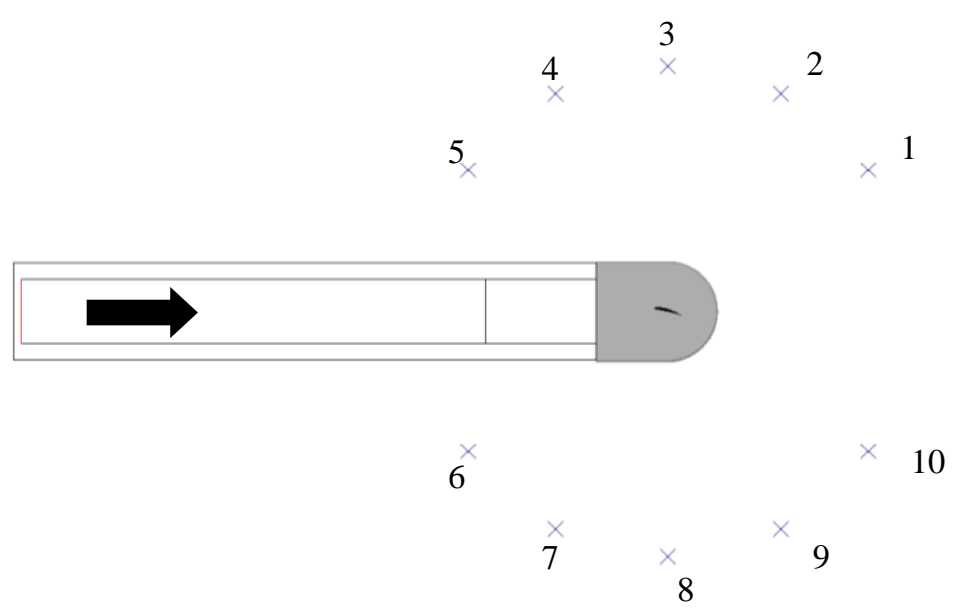

Figure 4. Acoustic pressure measurement locations.

\section{Validation and analysis}

\section{III.A. Tunnel flow quality}

Mean and fluctuating velocity profiles across the jet and the Boundary Layer (BL) are compared between simulation and experiment in Figure 5. Tunnel mean axis flow component $U / U_{0}$ is shown across the BL in Figure 5a). Experiment and simulation both possess a similar boundary layer thickness as illustrated by the two curves both reaching $99 \% U / U_{0}$ at $z=18 \mathrm{~mm}$ or $z / c=9 \%$.

The distribution of fluctuations in the boundary layer is shown in Figure 5b). The maximum value of the experimental velocity fluctuation $u / U_{0}$ of $8 \%$ is greater than the simulation maximum of $6.2 \%$, calculated from turbulent kinetic energy, but the overall boundary layer shape is well captured away from the wall.

Based on these two results, the boundary layer topology in the simulation is very close to the experimental conditions, providing the necessary ground for an accurate study of the tip flow. 


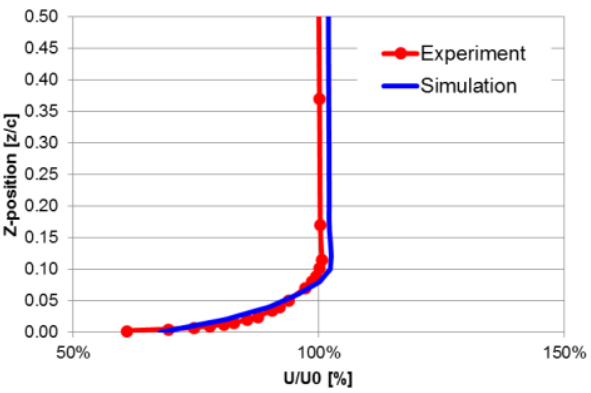

a)

Figure 5. Mean (a) and fluctuating (b) streamwise velocity magnitude normalized by maximum experimental mean velocity $U_{o}$ near the gap. (-) Experimental data; (-) simulation results.

\section{III.B. Mean pressure and velocity}

$C_{P}$ values for the baseline case are shown in Figure 6. Figure 6a) shows the $C_{P}$ profile at $z=110 \mathrm{~mm}$, near the mid-span, and Figure 6b) shows the $C_{P}$ at $z=11.5 \mathrm{~mm}$, near the tip gap located between $z=0 \mathrm{~mm}$ and $z=10 \mathrm{~mm}$. Figure 6a) shows a pressure profile with a leading edge pressure peak, characteristic of an airfoil in a 2D flow. Installation effects reduce the effective AoA in the baseline configuration from $15^{\circ}$ to $\sim 7^{\circ}$. The mid-span simulated $C_{P}$ values are within $1-2 \%$ the experimental measurements.

For the near gap flow locations at $z=11.5 \mathrm{~mm}, C_{P}$ magnitude is reduced as shown in Figure $6 \mathrm{~b}$ ) both by the reduced width of the leading edge pressure peak from $2 \%$ to $5 \%$ chord and by the reduction in magnitude of minimum pressure from $C_{P}=-1$ to $C_{P}=-0.75$. At $z=11.5 \mathrm{~mm}$, the three peaks in $C_{P}$ on the suction side correspond to time averaged tip leakage vortex structures as illustrated in Figure 7. The simulated mean $C_{P}$ values within 5\% of experiment shows that the mean pressures are converged to the correct values in the simulated time interval, meaning that the coherent structures of the flow have been correctly captured.
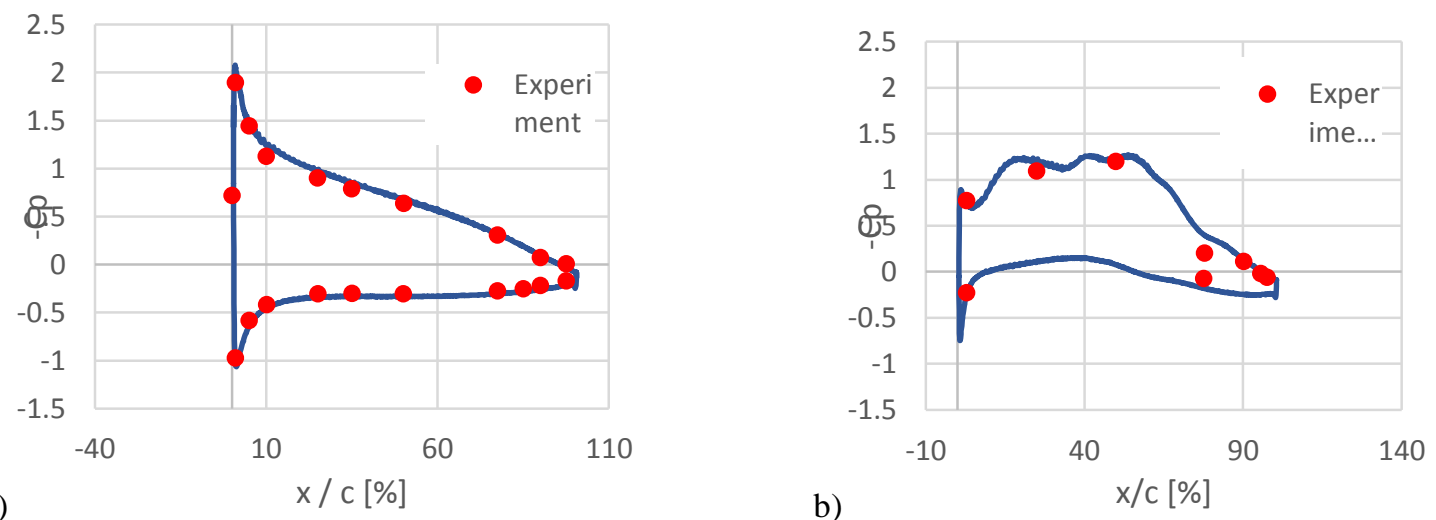

Figure 6. Mean pressure coefficient $C_{P}$ along the chord (baseline configuration). a) $z=110 \mathrm{~mm}$, near the airfoil mid-span. b) $z=11.5 \mathrm{~mm}$, near the tip gap. (•) Experimental data; (一) simulation results. 


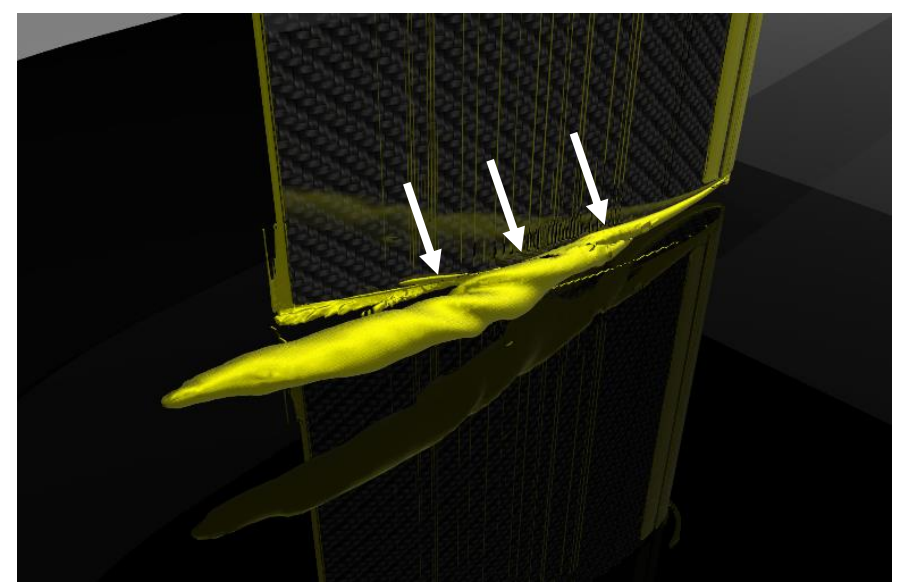

Figure 7. Mean coherent vortices rolling around the tip gap. Isosurface of time-averaged Lambda2 at $-1 \mathrm{e} 71 / \mathrm{sec}^{2}$.

Mean velocity components normalized by $U_{0}$ are plotted in the middle of the tip gap, at $\Delta z / 2=5 \mathrm{~mm}$, in Figure 7a) and $7 \mathrm{~b}$ ). The simulated flow in the gap presents qualitatively the same features as the experimental results. Near the gap, as shown in Figure 7b), the mean $x$-velocity is dominated by the tip vortex acceleration effect, which causes the strong mean $x$-velocity $>0.9\left|U_{0}\right|$ near the suction surface over the front of the airfoil, and the reduced $x$-mean velocity $<0.4\left|U_{0}\right|$ over the rear half of the airfoil.
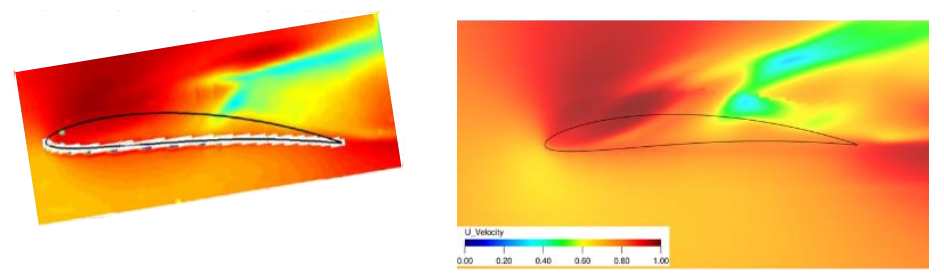

a) Streamwise direction dimensionless $x$-mean velocity component, $z=5 \mathrm{~mm}$
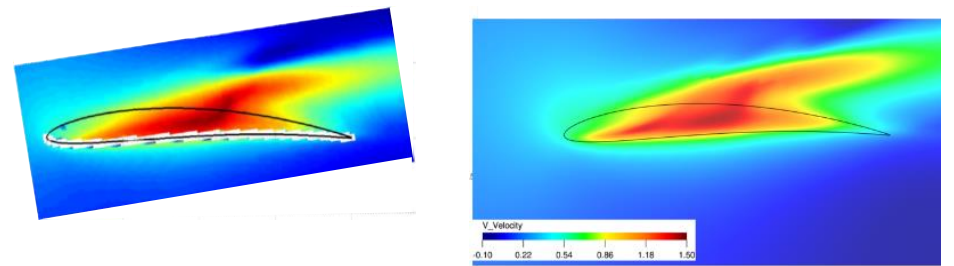

b) Normal direction dimensionless $y$-mean velocity component, $z=5 \mathrm{~mm}$

Figure 7. Mean velocity components adimensionalized by $U_{0}$ from PIV (left) and simulation (right).

\section{III.C. Near-gap wall pressure fluctuations}

In this section, the analysis focuses on unsteady flow features recorded on the airfoil surface. As this CFD/CAA LBM solver is by nature unsteady, time histories of pressure and velocity can be recorded at any location and any signal post-processing performed similarly to the experimental study ${ }^{1}$. The probe positions used for power spectrum density (PSD) comparison of the wall pressure fluctuation (WPF) are illustrated in Figure 2. The PSD of the WPF are plotted for experiment and simulation in Figure 8 following specific grouping: From 8a) to 8c), probes 24, 25 and 27 corresponding to the downstream section of the airfoil suction side near the gap and the trailing edge; from 8d) to 8f) probes 19, 20 and 21 corresponding to several points on the airfoil suction side along the tip gap; from 8g) to 8i) probes 51, 52 and B corresponding to points within the gap and in the flow directly downstream the gap.

At Probe 24, located on the suction side $16 \mathrm{~mm}$ inboard of the tip gap, the WPF levels are lower than $95 \mathrm{~dB}$, lower by $5-10 \mathrm{~dB}$ than all other probe locations, indicating a les turbulent floe. The simulated WPF matches the experimental 
broadband WPF from $100 \mathrm{~Hz}$ to $3 \mathrm{kHz}$, showing a broad peak value at $105 \mathrm{~dB}$ below 200Hz. Spectrogram plots (not presented here) show that the experimental WPF PSD varies by $+/-5 \mathrm{~dB}$ below $500 \mathrm{~Hz}$. At probe 25 , located at the corner of the trailing edge and the tip gap, $1.5 \mathrm{~mm}$ inboard, the flow is disturbed by the turbulent flow generated by the gap, with a broad peak between 1 and $2 \mathrm{kHz}$, captured in both experiment and simulation. At this probe, simulation and experiment are within $1-2 \mathrm{~dB}$ above $1 \mathrm{kHz}$. Some discrepancy between experiment and simulation is observed between 300 and $500 \mathrm{~Hz}$ which could be explained by the sensitivity of this location to the trailing edge flow development and the edge geometry, which is "ideal" in the simulation. At probe 27, located on the trailing edge 16 $\mathrm{mm}$ inboard of the tip gap, the simulation results are within $5 \mathrm{~dB}$ of the experiment within the range $300 \mathrm{~Hz}-5 \mathrm{kHz}$, still within the uncertainty range associated to the experimental measurements. However, below $300 \mathrm{~Hz}$, the experimental WPF are up to $10 \mathrm{~dB}$ above the simulation results. This difference is likely associated to larger structures, assumed stronger in the experiment due to discrepancies between simulation and test setup, such as installation effects (discussed in the next section) or geometry differences (trailing edge thickness, airfoil roughness).

a) Probe 24

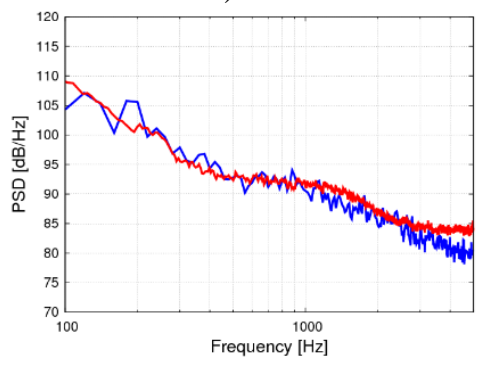

d) Probe 19

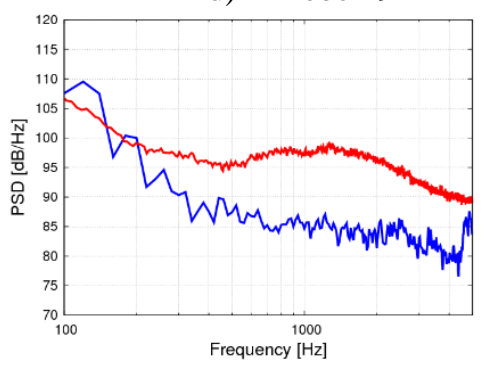

g) Probe 51

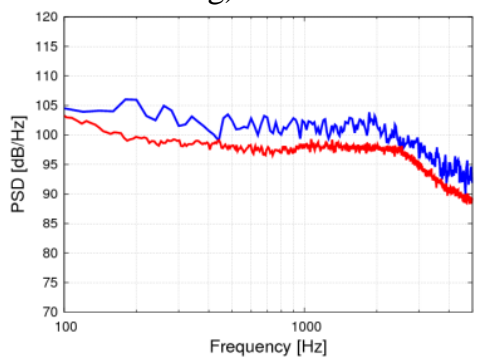

b) Probe 25

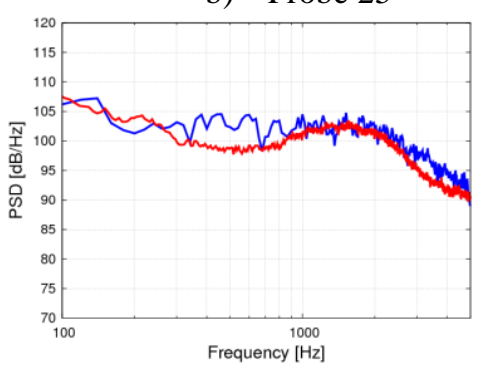

e) Probe 20

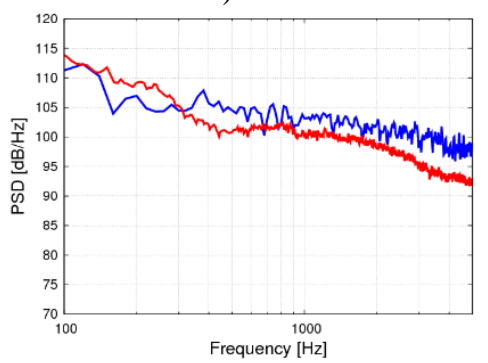

h) Probe 52

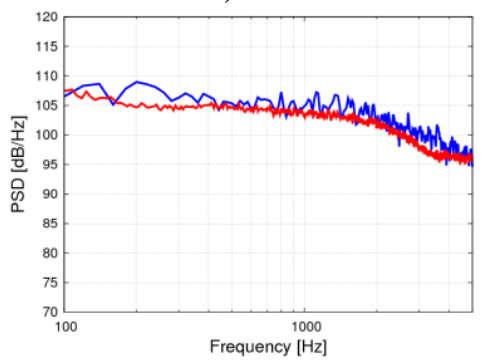

c) Probe 27

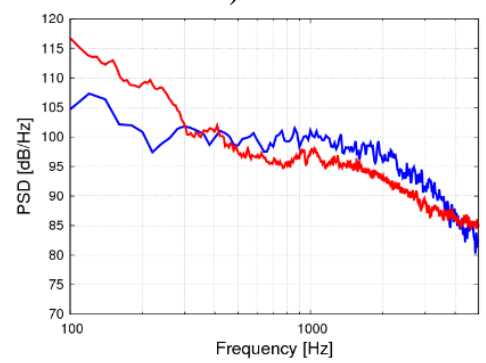

f) Probe 21

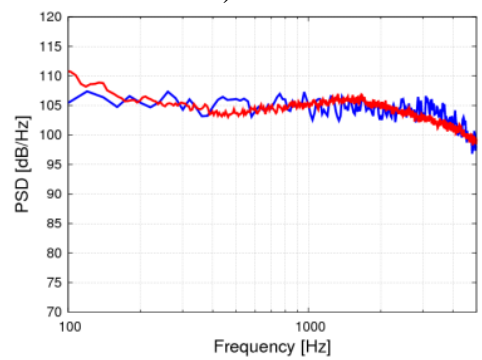

i) Probe B

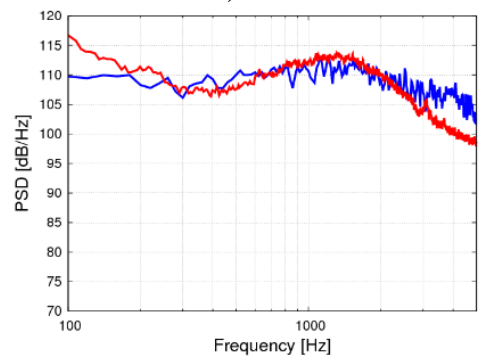

Figure 8. Pressure Power Spectrum Density (PSD) at various locations (see Figure 2). (一) Experimental data; (-) simulation results.

At Probe 19, the discrepancy between experiment and simulation results is between 10 and $15 \mathrm{~dB}$ above $200 \mathrm{~Hz}$. While the difference is above the $5 \mathrm{~dB}$ uncertainty of the experimental results, the probe location is in the vicinity of the separation point observed in Figure 7. Consequently, the observed WPF discrepancy shows that a 2-4 mm difference in the topology of the flow can strongly influence the results at a probe around the flow separation. Here the experimental probe is located 1-2 mm downstream the separation line, while the probe is located $1-2 \mathrm{~mm}$ upstream the separation line in the simulation, causing lower WPF in the simulation since the flow is laminar. At probe 20, the difference between the WPF results are back within the $+/-5 \mathrm{~dB}$ uncertainty range. While both in simulation and 
experiment the probe is located in the separation region, the observed discrepancy is likely related to the difference in the upstream flow with a higher coherence of the flow structures in the experiment. Finally, at Probe 21, further downstream the separation line, the experiment and simulation are within $2 \mathrm{~dB}$ for the frequency range $100 \mathrm{~Hz}-5 \mathrm{kHz}$. At this location, the flow is fully separated in both experiment and simulation, the coherent vortices start breaking up and the upstream separation topology does not influence as much the flow at the probe location.

A Probes 51 and 52, the WPF levels are within $4 \mathrm{~dB}$ over the whole frequency range, and the WPF curve shape matches the experimental results. These two probes illustrate again the importance of the flow topology on the WPF. While the distance between the two probes is less than $5 \mathrm{~mm}$, the flow separation happening on the airfoil suction side is different between simulation and experiment by less than $5 \mathrm{~mm}$, explaining the difference in WPF between test and simulation at Probe 51 of about 4-5 dB. At Probe 52, the probe is in the turbulent wake of the airfoil in both experiment and simulation, and then the WPF are within 2-3dB.

Finally, simulation and experiment are within $3 \mathrm{~dB}$ at Probe B, between $200 \mathrm{~Hz}$ and $3 \mathrm{kHz}$. Experiment WPF fall down quicker than simulation above $3 \mathrm{kHz}$ while the WPF level are up to $5 \mathrm{~dB}$ higher than simulation below $200 \mathrm{~Hz}$. Since the trends are inversed at low and high frequency, the experimental flow is likely more coherent, with larger structures and delayed turbulent eddy break up compared to simulated flow in the gap, causing the rise of WPF levels at low frequency and decrease at high frequency. However, the differences are within the uncertainty range and thus increase the confidence in the numerical method for solving these complex flow mechanisms and associated noise generation.

\section{III.D. Far-field noise}

In this section, the Sound Pressure Levels (SPL) measured in the simulation at the probe locations shown in Figure 4 are presented. While those results do not bring additional confidence in the method for capturing noise due to the absence of comparable experimental results at this time, they provide insights on the link between noise generation and surface WPF analyzing the correlation between WPF and Far-Field Noise (FFN) signals. In Figure 4, both SPL for the baseline configuration with $\Delta z=10 \mathrm{~mm}$ and no gap with $\Delta z=0 \mathrm{~mm}$ are presented to detect the effects of the tip flow. In Figure 3c), the baseline configuration is higher by $4 \mathrm{~dB}$ than the no gap configuration within the range 600 $\mathrm{Hz}-1 \mathrm{kHz}$. This bump can correlate to the tip flow effect, as a similar bump was observed in the $800 \mathrm{~Hz}-2 \mathrm{kHz}$ range on the WPF curves in Figure 8b) and 8i). However, this SPL bump is not observed in the other far-field probes. Consequently, this tip flow noise generation mechanism only radiates to the direction perpendicular to the flow, on the suction side. On all other SPL graphs, the bump is either absent or the difference with the no gap case is less than $2 \mathrm{~dB}$. However, differences in SPL levels between no gap and baseline configuration are observed on all other probes, with the no gap case SPL higher by $5 \mathrm{~dB}$ in the $200 \mathrm{~Hz}-600 \mathrm{~Hz}$ range at probes 4 to 7 . Other flow-induced noise mechanisms are present in the tip gap flow, which are responsible for higher SPL differences between no gap and baseline configurations. While the WPF are the characteristic pressure prints of the flow fluctuations on the surface, a direct correlation between WPF amplitude and FFN SPL cannot be drawn from the all curve shapes. The WPF curve shape of Probe 24 is the closest to the FFN SPL shape. However, Probe 24 is the furthest from the tip gap. Either the flow mechanisms in this area are the dominant source of noise or, more likely, the WPF at this location are an image of the noise generated in another location in the fluid. The parametric study in the next section, based on tip gap width and angle of attack will help at better understanding the noise generation mechanisms relative importance.

In Figure 10a), the noise sources distribution in the tip gap flow area is presented via an isosurface of Acoustic Power Volume Density calculated with the Flow-Induced Noise Detection (FIND) module. This module uses an innovative approach based on the dynamics of the flows structures and Alan Powell theoretical work on vortex sound ${ }^{34}$ to calculate the noise sources topology and strength. FIND has already been applied in the literature as a noise sources detection tool for HVAC systems noise ${ }^{35}$, wind noise ${ }^{36}$ and airframe noise ${ }^{37}$. In this figure, while some noise sources are located near the tip gap area, the major part of the noise sources are clearly located in the wake of the tip gap flow, where the eddy break-up of the merging tip vortices is happening as observed in Figure 10b). While the tip gap flow is leading to noise generation, a dominant broadband noise generation mechanism is the turbulent merging of the coherent tip vortices occurring downstream the tip gap flow. 
a) Probe 1

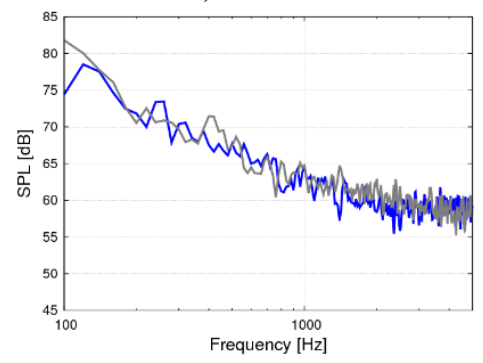

d) Probe 4

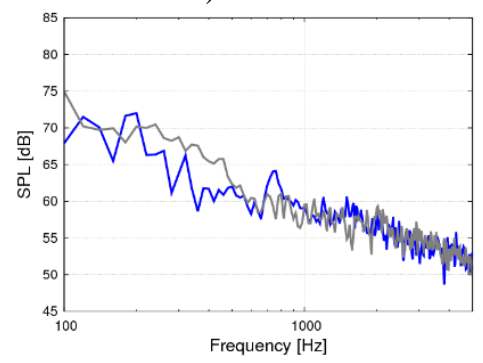

g) Probe 7

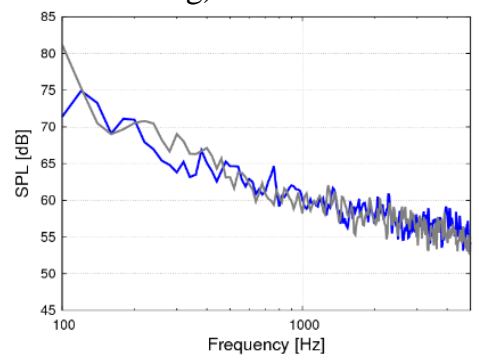

b) Probe 2

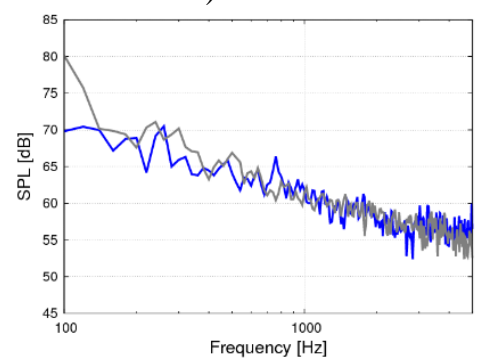

e) Probe 5

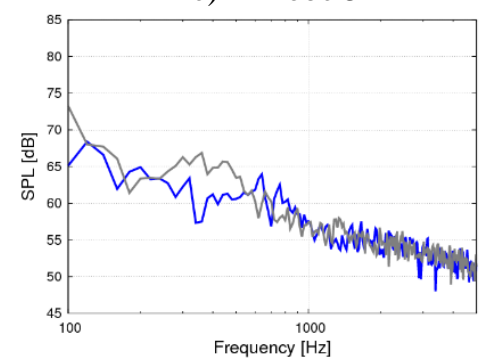

h) Probe 8

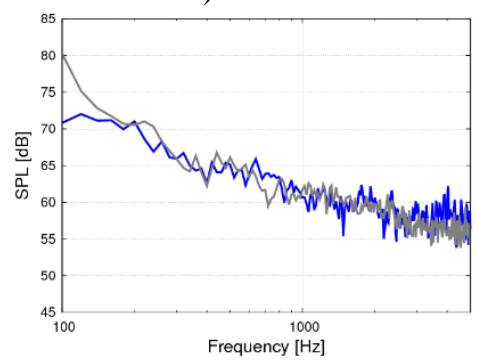

c) Probe 3

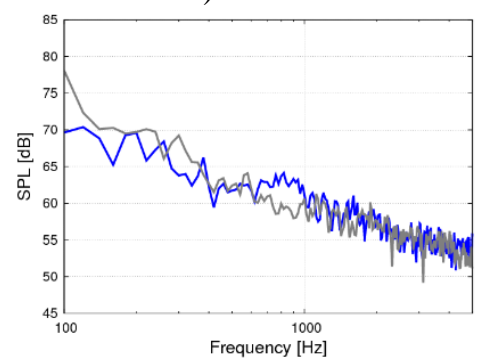

f) Probe 6

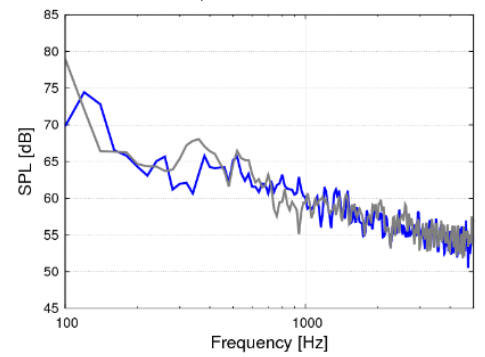

i) Probe 9

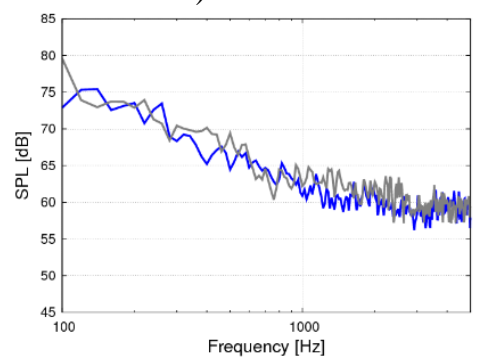

Figure 9. Sound Pressure Levels (SPL) at far field noise measurement locations (see Figure 3) directly measured in the LBM simulation. (-) no gap; (-) baseline (10mm gap)
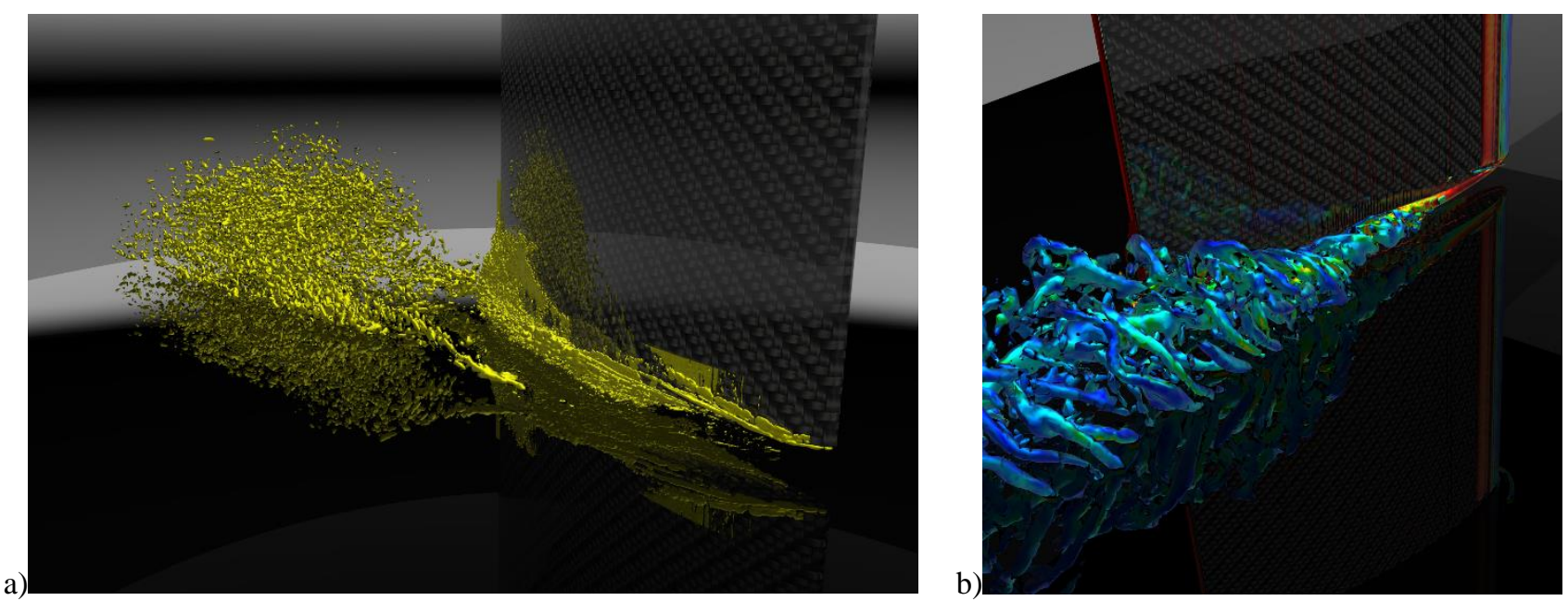

Figure 10. Broadband Noise Sources and flow topology:

a) FIND Acoustic Power isosurface at $\mathbf{- 1 0}$ dB[latticeVolume] between $500 \mathrm{~Hz}$ and $5 \mathrm{kHz}$;

b) Lambda2 isosurface at $-1 \mathrm{e} 7 \mathrm{1} / \mathrm{sec}^{2}$ colored by vorticity magnitude. 


\section{III.E. Installation effects analysis}

In this section, the analysis of the flow is focused on the installation effects related to the shear layer flow at the exit of the wind tunnel. While the WPF results at the surface of the airfoil were validated, the general surrounding flow must also be investigated. In addition to providing confidence in the simulation method, it brings additional insight on some of the discrepancies observed in the previous section.

The transient flow field at the mid-plane of the end plates is dominated by a periodically oscillating shear layer flow at the exit of the wind tunnel, at the top and bottom of the tunnel, as shown at $1 / 4 \mathrm{~T}, 1 / 2 \mathrm{~T}, 3 / 4 \mathrm{~T}$, and $\mathrm{T}$ for baseline configuration in Figure 11, where $\mathrm{T}$ is the period of the shear layer shedding. This phenomenon has potentially two negative effects on the investigation. First, the strong pressure fluctuations associated to the shear layer fluctuations can artificially trigger an early detachment on the airfoil side. This phenomenon was for example observed on preliminary coarser runs, where the shear layer flow was artificially amplified, resulting in a strong coherent flow oscillation and a periodic early detachment on the suction side of the airfoil. Because this shedding changes the topology of the flow and increases the turbulence of the airfoil wake, WPF and FFN SPL levels change. Second, this coherent shear layer breaks up downstream the airfoil as observed in Figure 11, creating mid-to-high frequency noise sources potentially overpowering the noise sources in the tip gap flow, which is the focus of this study.

In the experiment, shear layer Kelvin-Helmholtz instabilities are present at the 2-D jet boundary in the $y$ direction. The dominant pressure fluctuation frequency is $52 \mathrm{~Hz}$ which corresponds to the main coherent jet shear layer instability. The $52 \mathrm{~Hz}$ fluctuations caused by the shear layer can be seen in the plot of WPF presented in Figure 12 and are overall dominant by 30-40 dB. Figure 12a) shows PSD of WPF at Probe 23 for the baseline case, $\Delta z=10 \mathrm{~mm}$, and for a case with angle of attack $A o A=18 \mathrm{deg}$. Same results for Probe 46 are presented in Figure 12b). The simulated fluctuation levels are higher than obtained experimentally by 5-6 dB, likely due to the idealized geometry of the wind tunnel and related differences in the incoming flow in the simulation. The PSD peak is also narrower than experiment, indicating a stronger coherent instability than in test.

While the results presented in the previous section were in good agreement with experiment, some differences were observed and are potentially related to this effect. However, while not presented in this study, the periodic detachment on the airfoil suction side caused by the shear layer was not observed in the final simulation results presented in this study. In order to improve the installation effects correlation between simulation and experiment, additional simulations could be performed with an increased resolution in the shear layer area. Since the focus of the study was on the tip gap flow, these additional results are not available at this time and shall be presented in future studies.

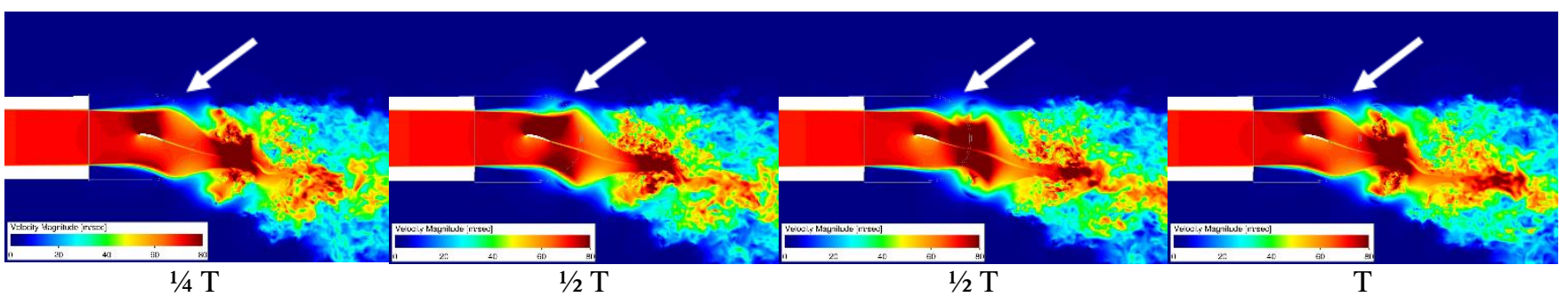

a) Streamwise direction velocity magnitude component, $z=5 \mathrm{~mm}$

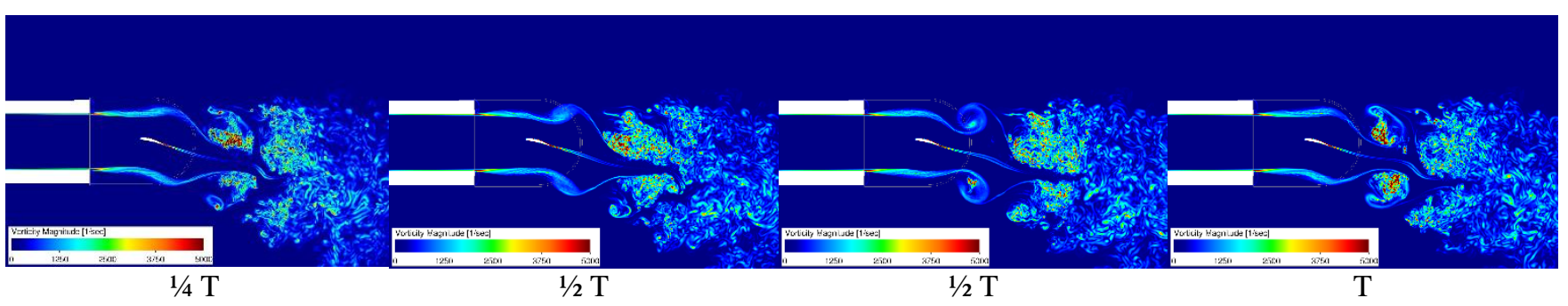

b) Streamwise direction vorticity magnitude component, $z=5 \mathrm{~mm}$

Figure 11. Installation effects: flow variables evolution between the plates. Arrow points at the shear layer shedding due to installation effects. 

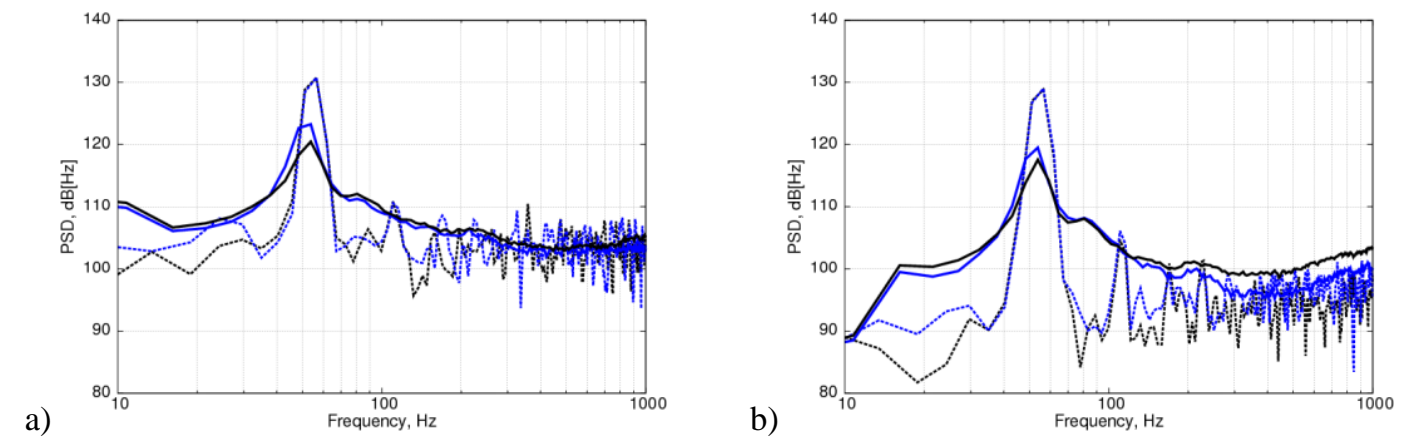

Figure 12. Pressure Power Spectrum Density at Probe 23 (a) and Probe 46 (b) for angles of attack $(\square) 15^{\circ} ;(\square) 18^{\circ}$ in the low frequency range $[10 \mathrm{~Hz}-1 \mathrm{kHz}]$.

(一) Experimental data; (---) simulation results.

\section{Parametric study}

To better understand the physical mechanisms of the tip gap noise and validate the conclusions from the previous section on the noise generation mechanisms, a parametric study is conducted with airfoil angle of attack $A o A=10^{\circ}$, $15^{\circ}$ and $18^{\circ}$, and tip gap width $\Delta z=0 \mathrm{~mm}, 5 \mathrm{~mm}$ and $10 \mathrm{~mm}$. The wind-tunnel mean flow speed is kept at $U_{0}=70 \mathrm{~m} / \mathrm{s}$, corresponding to $\operatorname{Re}_{\mathrm{c}}=9.6 \cdot 10^{5}$.

\section{IV.A. Tip gap width effects}

The tip gap fluctuations are visible in the WPF PSD spectra for probe 24, 25, 27, 20, 21 and 52, shown in Figure 13. As the tip gap thickness $\Delta z$ varies, the broadband content of the WPF changes, depending on the distribution of the probes over the tip gap area. For example, when $\Delta z=0 \mathrm{~mm}$, the WPF amplitude at probe 24 is higher by $5-10 \mathrm{~dB}$ than when the tip gap width is non-zero. This is explained looking at Figure 15a) where a detachment generating downstream-convected flow fluctuations is observed upstream probe 24. However, when the tip gap is present, the flow leakage creates coherent vortices which push back the flow towards the airfoil suction side, reducing the otherwise observed detachment and limiting the amount of fluctuations at probe 24. Similar conclusions can be drawn at probes 25 and 27, which are located near probe 24, and thus affected by this presence/absence of detachment. However, a non-linear effect of the tip gap width on the WPFis observed at Probe 25 as shown in Figure 13b). Both WPF amplitudes for $\Delta z=0 \mathrm{~mm}$ and $\Delta z=10 \mathrm{~mm}$ below $1 \mathrm{kHz}$ are higher by $5 \mathrm{~dB}$ than the WPF amplitudes for $\Delta z=5$ $\mathrm{mm}$. As the tip gap is created, the detachment on the suction side is first reduced in the vicinity of the tip and the WPF amplitudes are reduced broadband as observed in Figure 13b). As the tip gap width grows, tip vortices are generated, become instable and thus break-up in smaller structures, increasing the amplitude of the WPF. However, since the break-up happens in the coherent tip vortices away from the surface, the characteristic size of the eddies is larger than in a laminar-turbulent transition on an airfoil surface. Consequently, the high frequency content of the WPF which was observed at $\Delta z=0 \mathrm{~mm}$ is not present at $\Delta z=10 \mathrm{~mm}$ above $2 \mathrm{kHz}$. A similar behavior is also observed in Figure 13c) for Probe 27. The non-linear effect is observed, but, since its location is at the trailing edge, the $\Delta z=0 \mathrm{~mm}$ WPF amplitudes are only higher by $2-3 \mathrm{~dB}$ compared to the two other configurations.

As regards the probes located along the tip gap, the WPF amplitudes are directly correlated to the tip gap width change. As the tip gap grows, the WPF amplitudes along the tip gap increase. When the tip gap width is $\Delta z=0 \mathrm{~mm}$, the absence of tip gap flow removes the associated flow fluctuations along the tip gap, and the WPF amplitude is up to 30dB lower than when a tip gap is present as shown in Figure 13d) and 13f). For probes located further downstream, such as Probe 21, the effect of the tip gap absence is reduced as the probes are closer to the detachment region observed in Figure 15a). The $\Delta z=0 \mathrm{~mm}$ configuration is then not more than $10 \mathrm{~dB}$ lower than the two other configurations.

In addition, the noise generation follows the opposite trend compared to the flow fluctuations in the tip gap flow region. As observed in Figure $14 \mathrm{at} 1 \mathrm{kHz}$, the noise generation is strongest at $\Delta z=5 \mathrm{~mm}$, and lowest at $\Delta z=0 \mathrm{~mm}$. This is in agreement with the observations made on the baseline configuration. When $\Delta z=0 \mathrm{~mm}$, the tip leakage flow does not exist, and the break-up of the merging tip vortices does not happen. This is also confirmed by the absence of noise sources in the wake of the airfoil in Figure 16a). At $\Delta z=5 \mathrm{~mm}$, the fluctuations near the tip gap area were at the lowest of the 3 configurations. Consequently, the flow leakage generates highly coherent tip vortices, with high levels of vorticity which break up downstream the tip gap flow, interacting with the airfoil wake. This break up generates concentrated noise sources as observed in Figure 16b). As the tip gap width increases to $\Delta z=10 \mathrm{~mm}$, the tip leakage flow speed increases, and the generated tip vortices circulation increases while the distance between the vortices and the airfoil wake grows as observed in Figure 15c), when compared to $\Delta z=5 \mathrm{~mm}$ in Figure 15b). Consequently, the 
kinetic and turbulent energy of the flow is less concentrated and the eddy break up is of lesser intensity. The vortexvortex interactions in this region and the noise sources intensity are reduced, the sources distribution is diffuse, as observed in Figure 17c). This change in flow topology explains the lower FFN levels observed in Figure 14c) compared to Figure 14b).

In real conditions, with a rotating geometry, the effects of the tip leakage flow on the noise levels would be different than observed here, as the impingement of the generated eddies on the following blade would arguably become a dominant noise source generation mechanism.

a) Probe 24

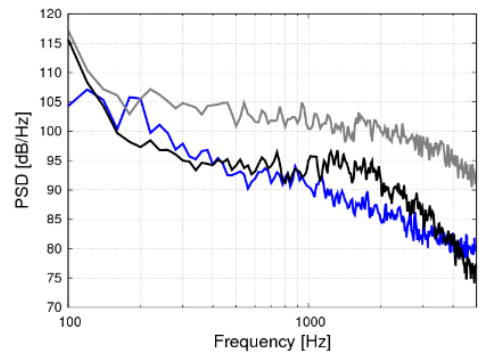

d) Probe 20

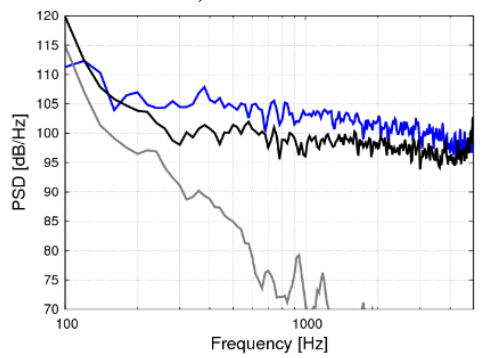

b) Probe 25

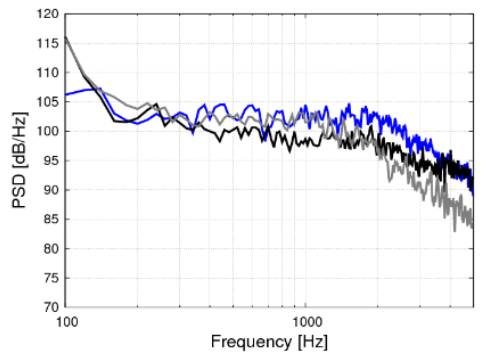

e) Probe 21

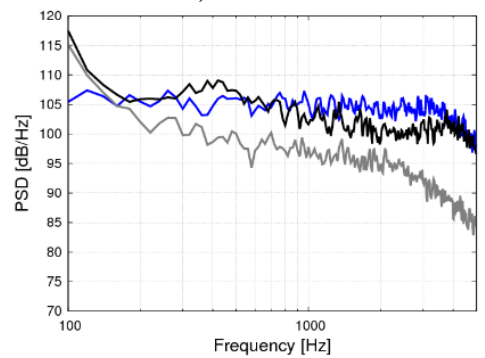

c) Probe 27

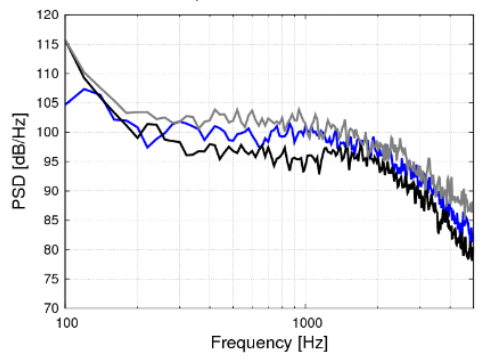

f) Probe 52

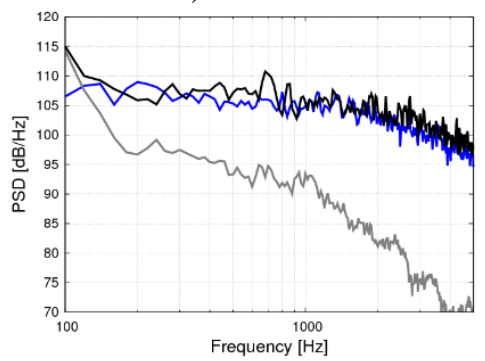

Figure 13. Simulated Pressure Power Spectrum Density at various locations (see Figure 2) for tip gap width (-) $0 \mathrm{~mm} ;(-) 5 \mathrm{~mm} ;(-) 10 \mathrm{~mm}$.
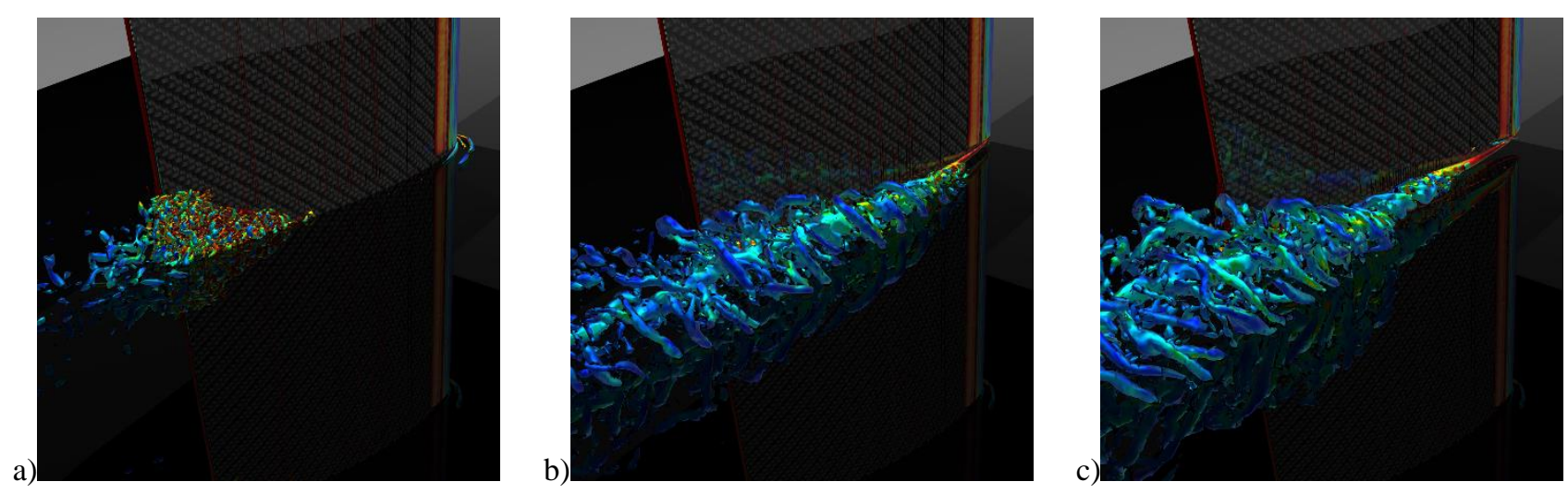

Figure 15. Gap size effects on tip vortices topology for tip gap widths of $0 \mathrm{~mm}(\mathrm{a}), 5 \mathrm{~mm}$ (b) and $10 \mathrm{~mm}$ (c): Lambda 2 isosurface at $-1 \mathrm{e} 7 \mathrm{1} / \mathrm{sec}^{2}$ colored by vorticity magnitude. 

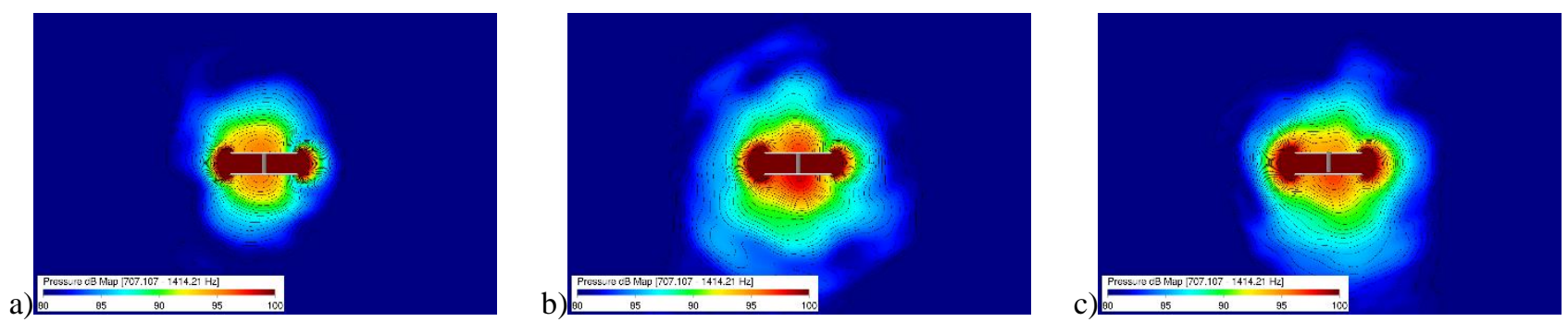

Figure 14. Gap size effects on pressure field amplitude for tip gap widths of $0 \mathrm{~mm}$ (a), $5 \mathrm{~mm}$ (b) and $10 \mathrm{~mm}$ (c): Pressure dB maps for octave frequency band centered on $1 \mathrm{kHz}$.

a)
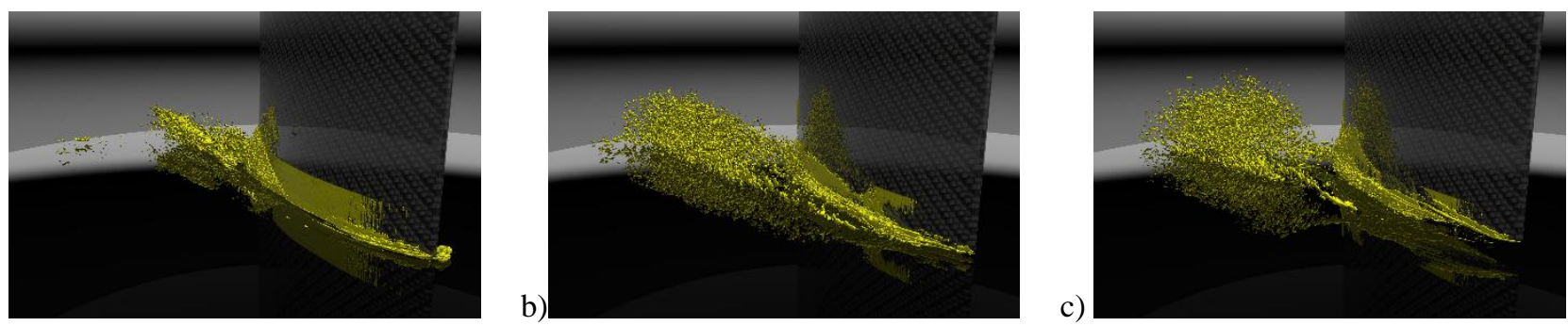

Figure 16. Broadband Noise Sources topology for tip gap width of $0 \mathrm{~mm}(\mathrm{a}), 5 \mathrm{~mm}$ (b) and $10 \mathrm{~mm}$ (c): FIND Acoustic Power isosurface at $-10 \mathrm{~dB}$ [latticeVolume] between $500 \mathrm{~Hz}$ and $5 \mathrm{kHz}$

\section{IV.B. Angle of attack effects}

The tip gap fluctuations are visible in the WPF PSD spectra for probe 24, 25, 27, 20, 21 and 52, shown in Figure 17. At all probes, the WPF for angle of attack $A o A=10^{\circ}$ are lower by 5 to $10 \mathrm{~dB}$ over the frequency range $100 \mathrm{~Hz}-5$ $\mathrm{kHz}$ than the WPF for $A o A=15^{\circ}$ and $A o A=18^{\circ}$. The WPF levels are directly connected to the pressure distribution on the suction side resulting from the angle of attack. As the angle of attack increases, the flow within the tip is accelerated and increases the tip vortices intensity. In addition, the pressure decreases on the airfoil suction side downstream the leading edge. At angle of attack $A o A=10^{\circ}$, the coherent tip vortex is pushed downstream the airfoil, with high speed convection flow happening along the suction side, as observed in Figure 18a). The flow structure breaks up downstream the airfoil, away from the surface probes. The WPF levels at the probes are thus at the lowest. When the angle of attack increases to $A o A=15^{\circ}$, the tip vortex becomes stronger as the flow within the tip gap accelerates. However, the velocity along the airfoil is lower as the pressure on the suction side decreases. An earlier break-up of the tip vortex consequently happens, as observed in Figure 18b) and the WPF levels are increased by 5 to $10 \mathrm{~dB}$ compared to $A o A=10^{\circ}$ as shown in Figure 17. As the angle of attack further increases up to $A o A=18^{\circ}$, the pressure on the suction side further decreases. This results in an even stronger break-up of the coherent tip vortices close to the airfoil surface as observed in Figure 18c). WPF levels are consequently increased by $0-2 \mathrm{~dB}$, as observed in Figure 17 on all probes, especially close to the trailing edge.

As regards noise levels, the correlation between WPF levels and FFN levels is a priori not possible. As the angle of attack goes from $A o A=10^{\circ}$ to $A o A=15^{\circ}$, the FFN levels at $1 \mathrm{kHz}$ are reduced in all spatial directions as illustrated in Figure 19a) and 19b). However, as the angle of attack goes from $A o A=15^{\circ}$ to $A o A=18^{\circ}$, the FFN levels are increased at $1 \mathrm{kHz}$ in all spatial directions as illustrated in Figure 19b) and 19c). In terms of WPF, PSD levels monotonically increase as the angle of attack increases. Consequently, no direct conclusions can be drawn from the WPF amplitude relation to the angle of attack. 
a) Probe 24

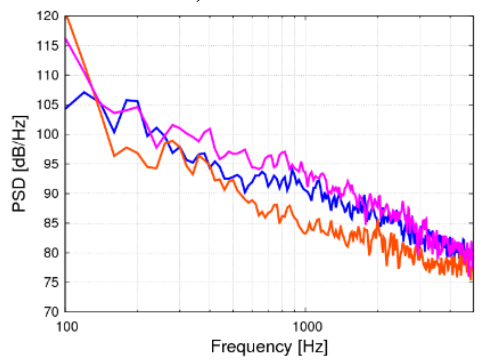

d) Probe 20

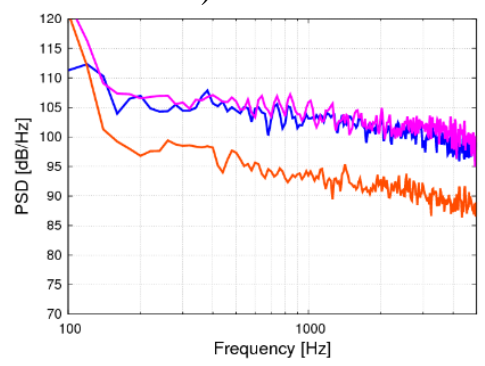

b) Probe 25

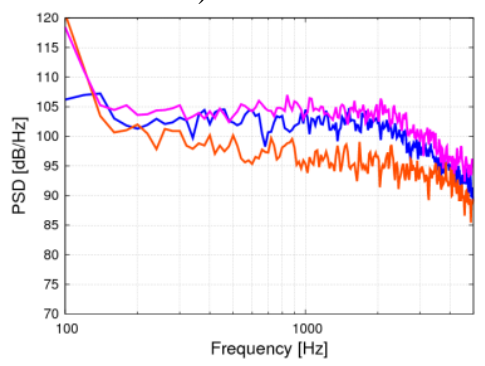

e) Probe 21

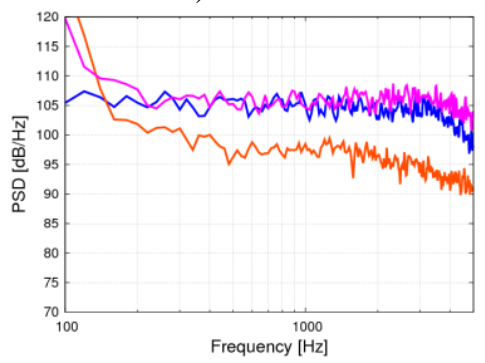

c) Probe 27

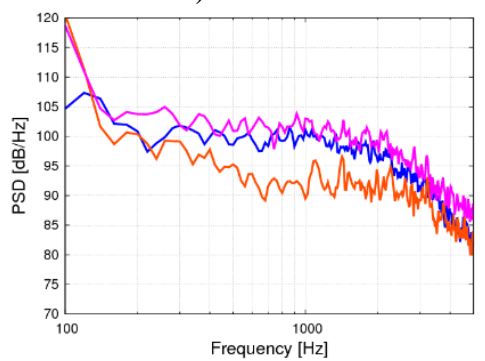

f) Probe 52

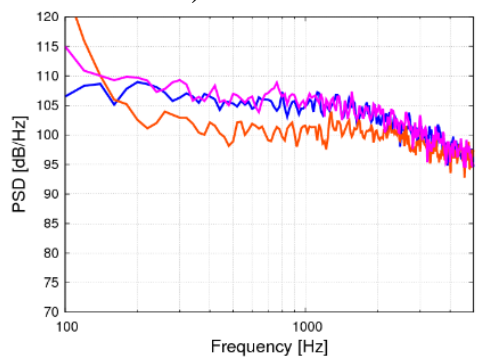

Figure 17. Simulated Pressure Power Spectrum Density at various locations (see Figure 2) for angle of attack $(\mathrm{AoA})(-) 10^{\circ} ;(-) 15^{\circ} ;(-) 18^{\circ}$.
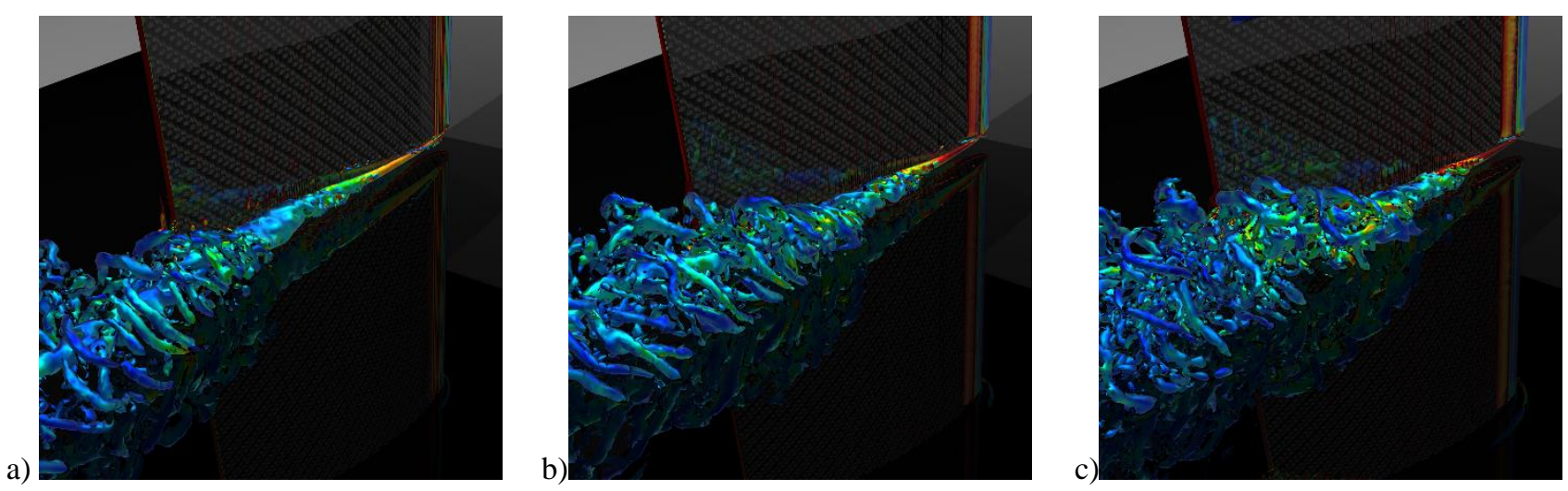

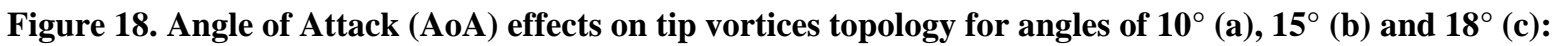
Lambda2 isosurface at $-1 \mathrm{e} 7 \mathrm{1} / \mathrm{sec}^{2}$ colored by vorticity magnitude.

Nevertheless, as discussed in the previous paragraph on tip gap width effects, the tip vortex flow varies in similar ways compared to the noise sources distribution and FFN noise levels. At $A o A=10^{\circ}$, the tip vortex coherent is pushed downstream and breaks up in a high velocity convection flow as observed in Figure 18a). Noise sources are consequently strong and concentrated as shown in Figure 20a). At $A o A=15^{\circ}$, the velocity on the suction side is reduced. The tip vortex breaks up in a less energetic convection flow as shown in Figure 17b). The noise sources are thus less concentrated as shown in Figure 19b), and possess lower noise levels. Finally, as the angle of attack increases to $A o A=18^{\circ}$, the velocity on the suction side further decreases. Consequently, the tip vortex generated by the flow leakage breaks up more intensively than with $A o A=15^{\circ}$. This behavior can be observed when comparing Figure $18 \mathrm{~b}$ ) and $18 \mathrm{c}$ ). Because the break up is more intense, the generated noise sources are stronger as observed between Figure $20 \mathrm{~b}$ ) and 20c), resulting in higher noise levels in the far field as observed between Figure 19b) and Figure 19c). 
a)

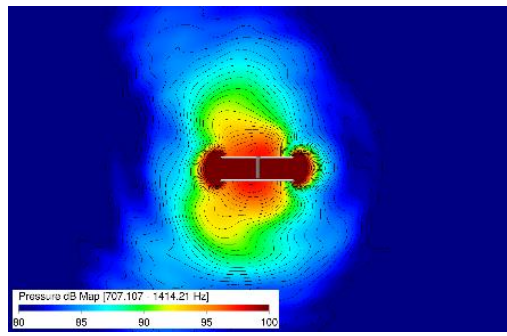

b)

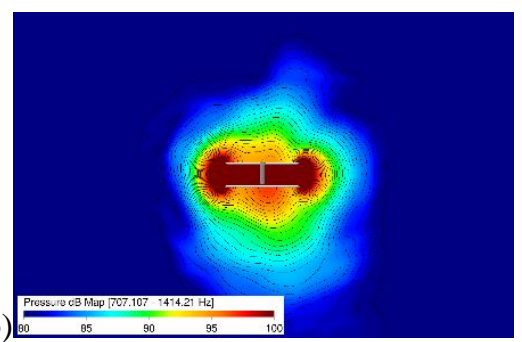

c)

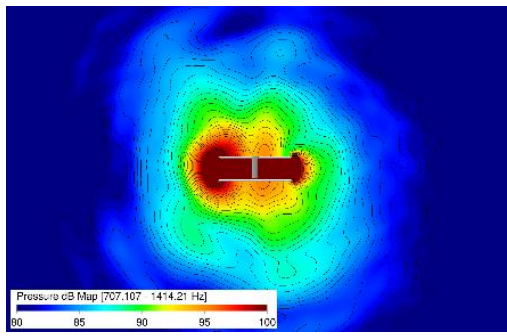

Figure 19. Angle of Attack (AoA) effect on acsoutic field amplitude for angles of $10^{\circ}(\mathrm{a}), 15^{\circ}(\mathrm{b})$ and $18^{\circ}(\mathrm{c})$ : Pressure dB maps for octave frequency band centered on $1 \mathrm{kHz}$.
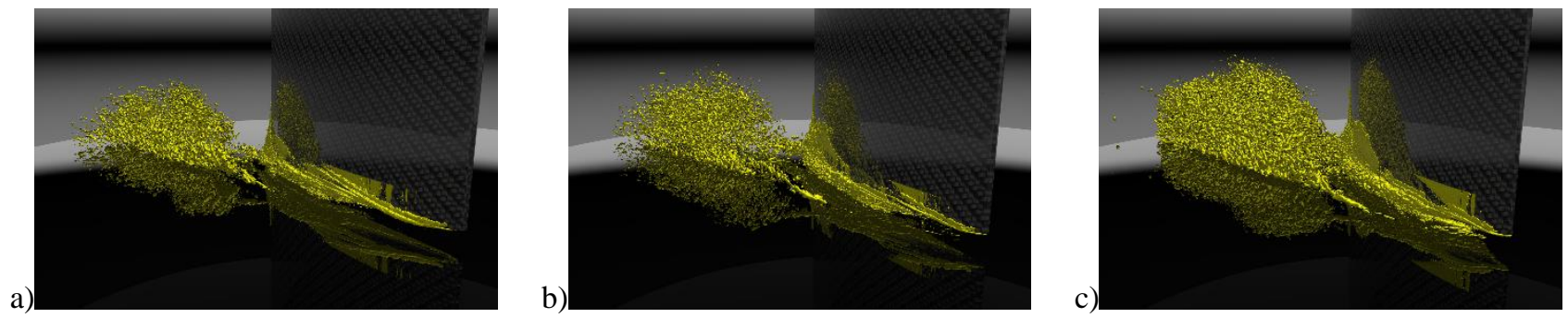

Figure 20. Broadband Noise Sources topology for angles of $10^{\circ}(\mathrm{a}), 1^{\circ}$ (b) and $18^{\circ}$ (c): FIND Acoustic Power isosurface at $\mathbf{- 1 0}$ dB[latticeVolume] between $500 \mathrm{~Hz}$ and $5 \mathrm{kHz}$

\section{Conclusions}

In this paper, an airfoil tip leakage simulation has been investigated and compared to experimental results. The simulation is performed using a LBM method and the comparison of the time averaged flow and WPF data on the airfoil surface shows very good agreement between simulation and experiment. The presented simulation tools can not only predict the noise generation of the tip gap, but also lead to a better understanding of the different noise generation mechanisms. The noise sources have been detected and directly linked to the coherent tip vortices break up interacting with the airfoil wake downstream and close to the airfoil. Mean fluctuating velocity components from the simulation near the tip gap confirm the existence of two main regions of fluctuations, first reported in the experimental analysis. Comparisons of wall pressure fluctuations show that the main pressure signals around the gap region have been recovered. Further analysis of the flow and noise sources topology provided unique insight on the flow mechanisms responsible for far-field noise levels, with the illustration of a limited correlation between WPF amplitude and far field noise levels, while a correlation between tip leakage flow topology and downstream flowinduced noise generation mechanisms was drawn. The $52 \mathrm{~Hz}$ pressure and velocity fluctuation peak noted in experiments as installation effects is also captured and identified in the simulation as the shear layer oscillation at the wind tunnel exit. The reported parametric study illustrates how the tip gap clearance and airfoil angle of attack impact the wall pressure fluctuations, as well as the far field noise levels and the noise sources topology and strength. The noise generation mechanisms were shown to be non-linearly correlated to the WPF amplitude as an increase in the WPF did not necessarily resulted in an increase of the noise levels. However, the WPF results were essential to understand the flow mechanisms in the tip gap area and how the evolution of the resulting tip vortical flow and its break up are impacted, with the corresponding generation of noise sources.

\section{References}

1 Grilliat, J., 2009, “Contribution a l'etude aeroacoustique des ecoulements de jeu”, PhD Thesis, Ecole Centrale de Lyon.

2 Grilliat, J., and Jacob, M. C, 2007, "Tip Leakage Experiment - Part One: Aerodynamic and Acoustic Measurements", 14th AIAA/CEAS. AIAA 2007-3684. 
3 Jacob, M. C., Grilliat, J., Camussi, R., and Gennaro, G. C., 2010, "Aeroacoustic investigation of a single airfoil tip leakage flow", International Journal of Aeroacoustics, Vol. 9-3, pp. 253-272.

4 Jacob, M. C., Grilliat, J., Roger, M., and Camussi, R., “Aeroacoustic Investigation of a Tip Clearance Flow”, 14th ICSV 2007.

5 Frisch, U., Hasslacher, B. and Pomeau, Y., 1986, “Lattice-gas Automata for the Navier-Stokes Equations”, Phys. Rev. Lett., Vol. 56, pp.1505-1508.

6 Chen, H., Teixeira, C. and Molvig, K., 1997, "Digital physics approach to computational fluid dynamics: Some basic theoretical features", Intl. J. Mod. Phys. C 9 (8), 675.

7 Guo, Z., Zhen, C. and Shi, B., 2002, "Discrete lattice effects on the forcing term in the lattice Boltzmann method", Phys. Rev. E, 65, 046308.

8 Li, Y., Shock, R., Zhang, R., and Chen, H., 2004, “Numerical Study of Flow Past an Impulsively Started Cylinder by Lattice Boltzmann Method" J. Fluid Mech., Vol. 519, pp. 273-300.

9 Shan, X. and Chen, H., 2007, "A general multiple-relaxation-time Boltzmann collision model", International Journal of Modern Physics C 18, 635

10 Shan, X. and Chen, H., 1993, "Lattice Boltzmann model for simulating flows with multiple phases and components", Phys. Rev. E, 47, 1815-1819.

11 Zhang, R., Shan, X. and Chen, H., 2006, "Efficient Kinetic Method for fluid simulation beyond Navier-Stokes equation", Phys. Rev. E, 74, 046703.

12 Thantanapally, C., Singh, S., Succi, S., and Ansumali, S., 2013, “Quasi-equilibrium lattice Boltzmann models with tunable Prandtl number for incompressible hydrodynamics", International Journal of Modern Physics C 24 (12), 1340004.

13 Moron, P., Powel, R., Freed, D., Pérot, F., Crouse, B., Neuhierl, B., Ullrich, F., Holl, M., Waibl, A., Fertl, C., 2009,"A CFD/SEA approach for prediction of vehicle interior noise due to wind noise", SAE 2009 Noise and Vibration Conference and Exhibition, 2009-01-2203.

14 Brès, G.A., Pérot, F., Freed, D., 2009, "Properties of the Lattice-Boltzmann Method for Acoustics", AIAA paper 2009-3395.

15 Casalino, D., Ribeiro, A., Fares, E., 2014, " Facing rim cavities fluctuation modes", Journal of Sound and Vibration 333 (2014) 2812-2830

16 Oettle, N., Meskine, M., Senthooran, S., Bissell, A., Balasubramanian, G., Powell, R., 2015,“A Computational Approach to Assess Buffeting and Broadband Noise Generated by a Vehicle Sunroof”, SAE World Congress, 201501-1532

17 Mann, A., Kim, M., Neuhierl, B., Perot, F. et al., 2015, "Exhaust and Muffler Aeroacoustics Predictions using Lattice Boltzmann Method", SAE Int. J. Passeng. Cars - Mech. Syst. 8(3):1009-1017, 2015, doi:10.4271/2015-012314.

18 Pérot, F., Kim, M. S., Moreau, S., Henner, M., Neal, D., 2010, “Direct aeroacoustic prediction of a low-speed axial fan", AIAA paper 2010-3887.

19 Pérot, F., Kim, M. S., Moreau, S., Neal, D., 2010, “Investigation of the flow generated by an axial 3-blade fan", 13th ISROMAC 2010-082.

20 Pérot, F., Kim, M. S., Meskine, M., Freed, D., 2012, "NREL wind turbine aerodynamic validation and noise predictions using Lattice Boltzmann Method", AIAA paper 2012-2290.

21 Mann, A., Pérot, F., Kim, M., Casalino, D., Fares, E., 2012, “Advanced Noise Control Fan Direct Aeroacoustics Predictions using a Lattice-Boltzmann Method", AIAA paper 2012-2287.

22 Pérot, F., Wada, K., Norisada, K., Kitada, M., Hirayama, S., Sakai, M., Imahigasi, S., Sasaki, N., 2011, “HVAC Blower Aeroacoustics Predictions Based on the Lattice Boltzmann Method", AJK2011-23018, AJK Conference, Hamamatsu, Japan.

23 Pérot, F., Meskine, M., Le Goff, V., Vidal, V., Gille, F., Vergne, S., Dupuy, F., 2012, "Flow-induced noise predictions of complete HVAC systems using Lattice-Botlzmann Method", SIA $7^{\text {th }}$ Automotive and Railway Comfort Conference 2012.

24 Aissaoui, A., Tupake, R., Bijwe, V., Meskine, M. et al., 2015, "Flow-Induced Noise Optimization of SUV HVAC System using a Lattice Boltzmann Method" SAE Int. J. Passeng. Cars - Mech. Syst. 8(3):1053-1062, 2015, doi:10.4271/2015-01-2323.

25 Bhatnagar, P., Gross, E. and Krook, M., 1954, “A model for collision processes in gases. I. small amplitude processes in charged and neutral one-component systems", Phys. Rev., vol.94, pp.511-525.

26 Chapman, S. and Cowling, T., 1990, "The Mathematical Theory of Non-Uniform Gases", Cambridge University Press. 
27 Chen, H., Chen, S., Matthaeus, W. H., 1992, "Recovery of the Navier-Stokes equations through a lattice gas Boltzmann equation method", Physical Review A, vol.45, 5339.

28 Chen, H., Kandasamy, S., Orszag, S., Shock, R., Succi, S. and Yakhot, V., 2003, “Extended Boltzmann Kinetic Equation for Turbulent Flows", Science, Vol. 301, pp. 633-636.

29 Yakhot, V., 2003, "Extended Boltzmann Kinetic Equation for Turbulent Flows", Science, Vol. 301, pp. 633-636

30 Chen, H., 1998, "Volumetric Formulation of the Lattice Boltzmann Method for Fluid Dynamics: Basic Concept", Phys. Rev. E, Vol. 58, pp. 3955-3963.

31 Chen, H., Orszag, S., Staroselsky, I. and Succi, S., 2004, "Expanded Analogy between Boltzmann Kinetic Theory of Fluid and Turbulence", J. Fluid Mech., Vol. 519, pp. 307-314.

32 Chen, H., Goldhirsh, I., Orszag, S., 2008, "Discrete rotational symmetry, moment isotropy, and higher order lattice Boltzmann models" J. Sci. Computing, Vol. 34, Iss. 1, pp 87-112.

33 Chen, H., Teixeira, C., Molvig, K., 1998, "Realization of Fluid Boundary Conditions via Discrete Boltzmann Dynamics", Intl J. Mod. Phys. C, Vol. 9 (8), pp. 1281-1292.

34 Powell, A. 1964 "Theory of vortex sound" Journal of the Acoustical Society of America, 36(1), pp. $177-195$.

35 Mann, A., Pérot, F., Meskine, M., Kim, M.-S., 2015, “Designing quieter HVAC systems coupling LBM and flowinduced noise source identification methods", $10^{\text {th }}$ FKFS-Conference, Progress in Vehicle Aerodynamics and Thermal Management

36 Senthooran, S., Powell, R., Choi, E.S., Cyr, S., 2015, “Computational prediction of interior noise for design variations on a simplified vehicle", Internoise 2015.

37 Rougier, T., Bouvy. Q., Casalino, D., Appelbaum, J., Kleinclaus, C., 2015, "Design of quieter landing gears through lattice-Boltzmann CFD simulations", AIAA 2015-3259. 